\title{
Umbilical cord-derived mesenchymal stem cells can inhibit the biological functions of melanoma A375 cells
}

\author{
WEI WANG ${ }^{1}, \mathrm{LI} \mathrm{LI}^{2}$, FEI CHEN ${ }^{2}$ and $\mathrm{YU} \mathrm{YANG}^{3}$ \\ ${ }^{1}$ Department of Pathology, West China Second University Hospital, \\ Sichuan University, Key Laboratory of Birth Defects and Related Diseases of Women and Children, \\ Ministry of Education; ${ }^{2}$ Laboratory of Pathology, West China Hospital, Sichuan University; \\ ${ }^{3}$ Department of Medical Oncology, Cancer Center of West China Hospital, \\ Sichuan University, Chengdu, Sichuan 610041, P.R. China
}

Received October 11, 2017; Accepted May 8, 2018

DOI: 10.3892/or.2018.6446

\begin{abstract}
Tumor tropism is an important property of mesenchymal stem cells (MSCs) that has been used in tumor-targeting therapies. However, the effects of MSCs on tumors remain controversial. The aim of the present study was to investigate the effects of MSCs on A375 melanoma cells. Umbilical cord-derived mesenchymal stem cells (UCMSCs) were co-cultured with A375 cells. MTT and Transwell assays were used to assess cell proliferation and invasion, while flow cytometry was performed to detect the apoptosis and the cell cycle distribution of A375 cells. The expression levels of kinases were assayed by western blotting and fluorescence analysis was conducted to detect cytoskeletal rearrangement. The results clearly indicated that UCMSCs could inhibit the proliferation, induce apoptosis and suppress the invasion of A375 cells. Mechanistic studies revealed decreased expression of several kinases (AKT, STAT3 and mTOR) and UCMSCs were also found to promote cytoskeletal rearrangement in A375 cells. These results confirmed that UCMSCs exert antitumor effects on melanoma A375 cells.
\end{abstract}

\section{Introduction}

Mesenchymal stem cells (MSCs) are multipotent progenitors that constitute a small proportion of many tissues, including bone marrow, umbilical cord, amniotic fluid, adipose tissue and fetal lung tissue (1). MSCs are described as fibroblast-like cells with the property of plastic adherence during in vitro culture. MSCs are able to differentiate into numerous cell lineages (such as adipocytes, osteoblasts, chondrocytes,

Correspondence to: Professor Yu Yang, Department of Medical Oncology, Cancer Center of West China Hospital, Sichuan University, 37 Guo Xue Xiang, Chengdu, Sichuan 610041, P.R. China

E-mail: yangyuflying@hotmail.com

Key words: mesenchymal stem cells, melanoma, A375, biological function, cytoskeletal rearrangement tenocytes and cells of visceral mesoderm) in the presence of different conditional media (2). MSCs are positive for the expression of CD105, CD73 and CD90 markers, and negative for the expression of the co-stimulatory factors CD34, CD80, CD86 and HLA-II (3). MSCs also have immunosuppressive capabilities, meaning that they can modulate the proliferation and function of all the components of the immune system. Increasing evidence indicates that MSCs inhibit the maturation of Tregs and dendritic cells and suppress the functions of memory and naïve $\mathrm{T}$ cells, as well as $\mathrm{CD} 4^{+}$and $\mathrm{CD} 8^{+} \mathrm{T}$ cells. Similar effects were also observed in $\mathrm{B}$ cells and natural killer cells (4).

To date, MSCs are considered to be optimal candidates for use in cell therapy based on these advantages. The in vivo immunomodulatory properties of MSCs play an important role in the maintenance of peripheral tolerance and the induction of transplantation tolerance, which can protect solid-organ grafts from being rejected (5). Transfusion of MSCs in systemic lupus erythematosus patients has been revealed to improve the levels of serological markers and stabilize renal function without the occurrence of serious adverse events (6). In addition, increasing evidence indicates that MSCs are involved in various diseases, such as type I diabetes (7), liver cirrhosis (8), cerebral palsy (9) and other autoimmune diseases (10). Collectively, these studies indicate that MSCs have potentially significant clinical applications.

However, the effect of MSCs on malignant tumors has remained controversial. Certain studies have indicated that MSCs could inhibit breast tumors (11) and lung cancer (12), whereas other studies have demonstrated that MSCs promote the metastasis of pancreatic (13) and prostate cancer cells (14). In the present study, MSCs isolated from umbilical cord were co-cultured with melanoma A375 cells, and the effect of MSCs on the function of A375 cells was investigated.

\section{Materials and methods}

Cell culture. The UCMSCs were purchased from the Sichuan Umbilical Cord Blood Stem Cell Bank (Chengdu, China). Following dissolving in a $37^{\circ} \mathrm{C}$ water bath, UCMSCs were maintained in Dulbecco's modified Eagle's medium (DMEM; 
Table I. List of primers in quantitative PCR detection.

\begin{tabular}{llll}
\hline Gene & \multicolumn{1}{c}{ Forward primer } & \multicolumn{1}{c}{ Reverse primer } & \multicolumn{1}{c}{ GenBank number } \\
\hline BCL2 & CAGGTTATATCTCATCTTTGAG & GTTGAGTAACGAGCTGACCCC & KY098794 \\
Survivin & ACCATAACCCACCACAGC & CAGTTCGTCCCTTTCCAG & DQ508249 \\
MCL1 & ATTCCAGAACAGGAGTACAGCTGT & CAGATGTACCCCTTGTGTAGAGT & NM_021960 \\
Bax & GACTTGAGTTGGGAGGGGAA & GAGGCTCAGCGCCAGGGCTGGG & KJ890756 \\
Fas & TGGCAACGCTGTCCTGTG & CCTTTTGCCAGTAGATGCGAG & KR709619 \\
TNF $\alpha$ & GGTGCTTGTTCCTCAGCCTC & CAGGCAGAAGAGCGTGGTG & M10988 \\
GAPDH & GAAGGTGAAGGTCGGAGTC & GAAGATGGTGATGGGATTTC & J04038 \\
\hline
\end{tabular}

BCL, B-cell lymphoma; MCL, myeloid cell leukemia; TNF, tumor necrosis factor; GAPDH, glyceraldehydes-3-phosphate dehydrogenase.

Invitrogen, Carlsbad, CA, USA) supplemented with $10 \%$ fetal bovine serum (FBS; Invitrogen) at $1 \times 10^{5}$ cells/well in a 6 -well plate (BD Falcon; BD Biosciences, Bedford, MA, USA). The medium was changed every two days, and adherent cells were harvested after two weeks using $0.25 \%$ trypsin (Gibco; Thermo Fisher Scientific, Inc., Waltham, MA, USA). Only UCMSCs passaged $<6$ times were used for co-culture since in our previous study (15) we observed that the differentiation of UCMSCs increased dramatically when they were passaged more than 6 times. Melanoma cells A375 [obtained from the American Type Culture Collection (ATCC; Manassas, VA, USA)] were cultured in RPMI-1640 (Gibco; Thermo Fisher Scientific) with $10 \%$ FBS. The co-culture ratio of UCMSCs to A375 cells was 2:1.

Cell proliferation assay. Cell proliferation was assessed by an MTT assay at 24, 48 and $72 \mathrm{~h}$ following co-culture.

Analysis of apoptosis and cell cycle distribution by flow cytometry. An apoptosis assay was performed by incubating the cells with $3 \mu \mathrm{l}$ Annexin V-FITC (Beyotime Institute of Biotechnology, Beijing, China) for $10 \mathrm{~min}$ and then counterstaining with $5 \mu \mathrm{l}$ PI, and detecting the apoptotic cells by flow cytometry. The cell cycle distribution of the A375 cells was also assessed by flow cytometry.

RNA extraction and quantitative PCR. Total RNA was extracted from A375 cells using an RNeasy Mini kit (Qiagen, Dusseldorf, Germany) at 24, 48 and $72 \mathrm{~h}$ post co-culture with UCMSCs. cDNA was synthesized using a ReverTra Ace qPCR RT kit (FSQ-101; Toyobo Co., Ltd., Kagoshima, Japan) with the following transcription conditions: $65^{\circ} \mathrm{C}(5 \mathrm{~min}), 37^{\circ} \mathrm{C}(15 \mathrm{~min})$ and $98^{\circ} \mathrm{C}$ (5 min). Real Master Mix (SYBR Green; FP202; Tiangen Biotech, Beijing, China) was used for the qPCR with an iCycler $\mathrm{iQ}^{\mathrm{TM}}$ Optical Module (Beckman Coulter, Fullerton, CA, USA) under the following conditions: $95^{\circ} \mathrm{C}$ for $30 \mathrm{sec}$, followed by 40 cycles at $95^{\circ} \mathrm{C}$ for $30 \mathrm{sec}, 58^{\circ} \mathrm{C}$ for $30 \mathrm{sec}$ and $72^{\circ} \mathrm{C}$ for $30 \mathrm{sec}$. A melt curve analysis followed, consisting of increasing temperatures from 55 to $95^{\circ} \mathrm{C}$ in $0.5^{\circ} \mathrm{C}$ increments at 10 -sec intervals for 40 cycles. The primers used are listed in Table I. All amplifications were performed three times.

Invasion assay. An invasion assay was performed using 24-well (8- $\mu \mathrm{m}$ pore size) Transwell plates (Corning, Lowell,
MA, USA). The A375 cells were plated in the upper chambers, which were pre-coated with Matrigel (20\%; BD Biosciences, Sparks, MD, USA), while UCMSCs were maintained in the bottom chamber. Toluidine blue (Leagene, Beijing, China) was used to stain the invaded A375 cells at 24 and 48 h post co-culture.

Western blot analysis. Collected A375 cells were washed twice with cold PBS and proteins were extracted using a protein extraction reagent (Pierce, Rockford, IL, USA) containing protease inhibitors (Roche Applied Science, Indianapolis, IN, USA). Protein concentration was assessed using the Micro BCA Protein Assay kit (Pierce). Protein samples $(20 \mu \mathrm{g})$ were separated by $12 \%$ SDS-polyacrylamide gel electrophoresis and transferred onto nitrocellulose membranes (Invitrogen). Following blocking, the membranes were incubated with primary antibodies (Table II) followed by horseradish peroxidase-conjugated secondary antibodies (1:5,000; cat. no. ab97040; Abcam, Cambridge, UK). Antigen-antibody complexes were visualized using an enhanced chemiluminescence reagent (Amersham Biosciences, Fairfield, CT, USA).

Fluorescence detection. A375 cells were maintained on glass slides and co-cultured with UCMSCs for $48 \mathrm{~h}$. Primary antibodies against E-cadherin and vimentin were applied to the cells for $4 \mathrm{~h}$ at room temperature. Following secondary antibody staining, A375 cells were viewed under a confocal scanning microscope (Carl Zeiss, Jena, Germany)at 488 and $525 \mathrm{~nm}$.

Statistical analysis. qPCR and western blotting results were analyzed with Bio-Rad CFX manager software (Bio-Rad Laboratories, Inc., Hercules, CA, USA) and Image Lab software 3.0 (Bio-Rad Laboratories), respectively. Cell cycle distribution was analyzed by ModFit 3.2 (Verity Software House, Topsham, ME, USA). All data are expressed as the mean \pm standard error using Student's t-test. SPSS 19.0 (IBM SPSS Statistics, Armonk, NY, USA) was used for statistical analysis. Values of $\mathrm{P}<0.05$ and $\mathrm{P}<0.01$ were considered to indicate statistically significant differences compared with the control group. All figures were generated with GraphPad Prism 5 (GraphPad Software, Inc., La Jolla, CA, USA). 
A

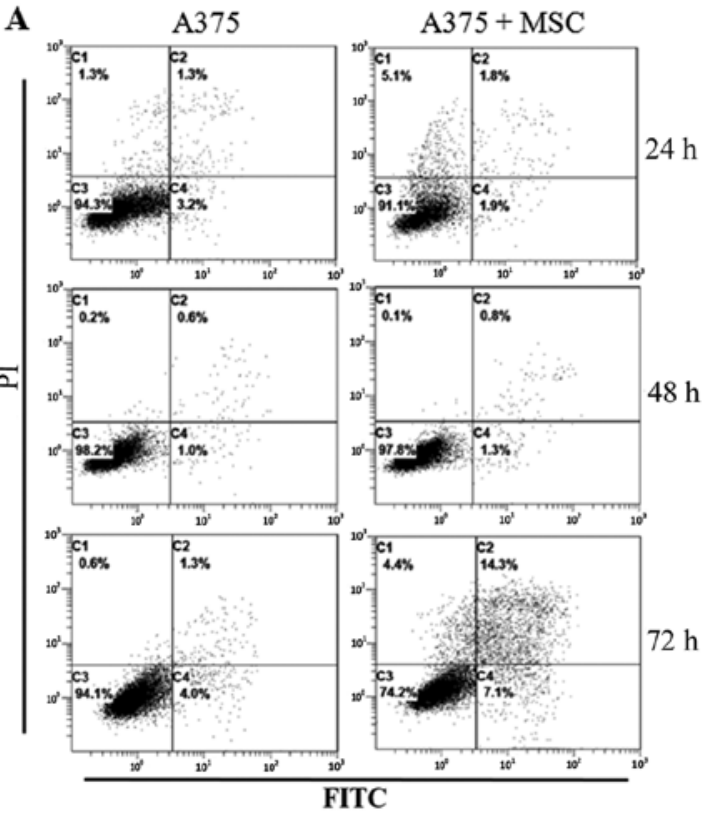

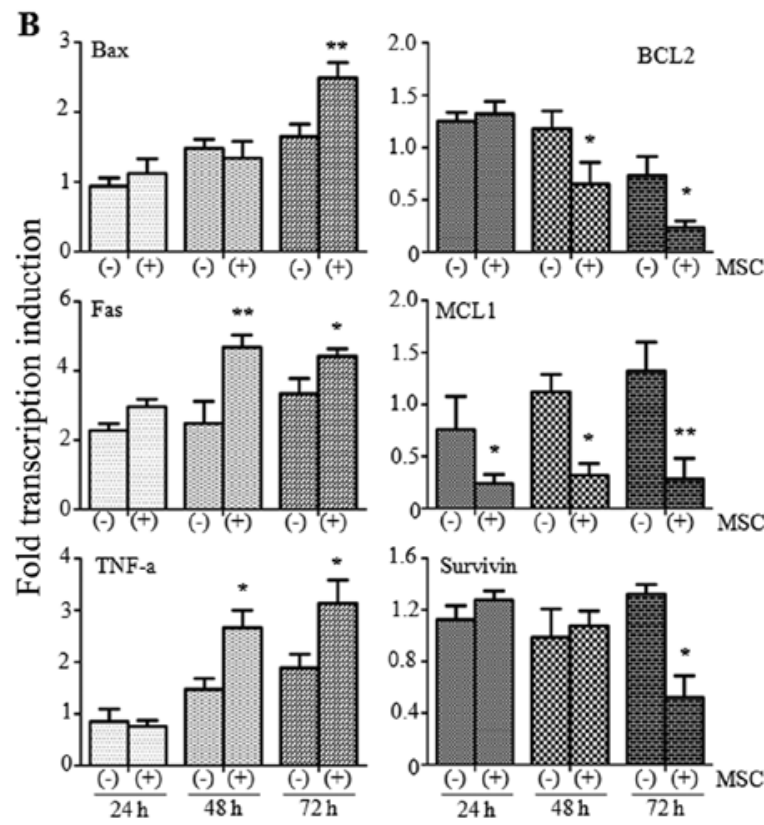

Figure 1. The apoptosis detection of A375 cells co-cultured with UCMSCs. (A) Flow cytometry for the apoptosis assay. (B) Real-time PCR detection of apoptosis-associated molecules. ${ }^{*} \mathrm{P}<0.05,{ }^{* *} \mathrm{P}<0.01$ vs. the control. UCMSCs, umbilical cord-derived mesenchymal stem cells.

Table II. The antibodies used in western blot analysis.

\begin{tabular}{lll}
\hline Antibody (dilutions) & Catalogue number & \multicolumn{1}{c}{ Company } \\
\hline AKT $(1: 1,000)$ & AF6261 & Affinity Biosciences \\
p-AKT $(1: 1,000)$ & AF0016 & Affinity Biosciences \\
PI3K $(1: 500)$ & AF6242 & Affinity Biosciences \\
p-PI3K $(1: 500)$ & AF3241 & Affinity Biosciences \\
STAT3 $(1: 1,000)$ & AF6294 & Affinity Biosciences \\
P-STAT3 $(1: 1,000)$ & AF3294 & Affinity Biosciences \\
ERK $(1: 1,000)$ & AF0155 & Affinity Biosciences \\
P-ERK $(1: 1,000)$ & AF1015 & Affinity Biosciences \\
MTOR $(1: 1,000)$ & AF6308 & Affinity Biosciences \\
P-MTOR $(1: 1,000)$ & AF3310 & Affinity Biosciences \\
GAPDH $(1: 1,000)$ & 200608 & Zen BioScience \\
\hline
\end{tabular}

aAffinity Biosciences, Zhenjiang, China; ' $Z$ Zen BioScience Co., Ltd., Chengdu, China.

\section{Results}

Apoptosis of A375 cells can be induced by UCMSCs. Flow cytometry was performed to detect the occurrence of apoptosis in A375 cells co-cultured with UCMSCs. The results indicated no obvious variation in the apoptotic rate between the A375 monoculture and A375+MSC co-culture groups at $24 \mathrm{~h}$ (4.5 vs. $3.7 \%$, respectively) or $48 \mathrm{~h}$ (1.6 vs. $2.1 \%$, respectively). However, the apoptotic rate increased markedly in the A375+MSC co-culture group (21.4\%) compared with the A375 monoculture group (5.3\%) at $72 \mathrm{~h}$ (Fig. 1A).

qPCR was then used to detect the levels of apoptosis-associated molecules in the different groups. Among the apoptosis-promoting factors, the expression of Bax was found to be increased at $72 \mathrm{~h}(\mathrm{P}<0.05)$ while Fas was markedly increased at $48 \mathrm{~h}(\mathrm{P}<0.01)$ and $72 \mathrm{~h}(\mathrm{P}<0.05)$ post co-culture compared with the A375 monoculture group. TNF- $\alpha$ was also found to be increased at 48 and $72 \mathrm{~h}$ (both $\mathrm{P}<0.05$ ) in the presence of UCMSCs. We also investigated three anti-apoptotic molecules: BCL2 was suppressed at 48 and $72 \mathrm{~h}$ (both $\mathrm{P}<0.05)$ while the expression of MCL1 was inhibited at $24 \mathrm{~h}$ $(\mathrm{P}<0.05), 48 \mathrm{~h}(\mathrm{P}<0.05)$ and $72 \mathrm{~h}(\mathrm{P}<0.01)$ post co-culture. Survivin was only decreased after $72 \mathrm{~h}(\mathrm{P}<0.05)$ (Fig. 1B). Our results indicated that UCMSCs could induce apoptosis in A375 cells.

UCMSCs inhibit the proliferation of A375 cells, however, have no effect on the cell cycle. Subsequently, we detected whether the proliferation and the cell cycle of A375 cells could be influenced by UCMSCs. In the MTT assay, the proliferation did not significantly differ between the A375 monoculture and the A375+UCMSCs co-culture groups at 24 and $48 \mathrm{~h}$. However, the proliferation of A375 cell was markedly inhibited $(\mathrm{P}<0.01)$ at $72 \mathrm{~h}$ post co-culture with UCMSCs (Fig. 2A). Flow cytometry was then used to assess the cell cycle distribution in various groups of A375 cells. The results indicated that UCMSCs had no effect on the cell cycle at 48 or $72 \mathrm{~h}$ post co-culture (Fig. $2 \mathrm{~B}$ and C).

UCMSCs suppress the invasive ability of A375 cells. As invasion is another important property of malignant tumor cells, we investigated the influence of UCMSCs on the invasive ability of A375 cells in a Transwell chamber pre-coated with Matrigel. As displayed in Fig. 3, the invaded cell numbers showed no obvious difference between the A375 and A375+MSC groups at $24 \mathrm{~h}$ post co-culture. However, the invasion of the A375 cells was significantly suppressed in the presence of UCMSCs after $48 \mathrm{~h}$ of co-culture. 


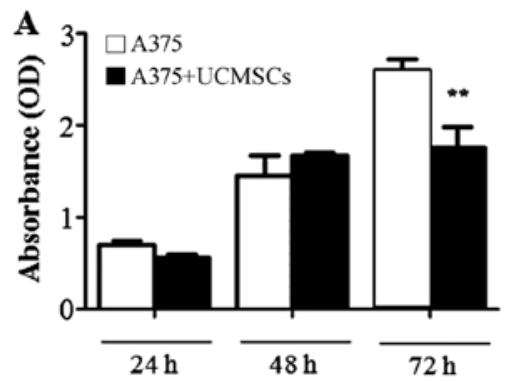

C
\begin{tabular}{|c|c|c|c|c|}
\hline & \multicolumn{2}{|c|}{$48 \mathrm{~h}$} & \multicolumn{2}{c|}{$72 \mathrm{~h}$} \\
\hline & A 375 & A $375+$ MSC & A 375 & A $375+$ MSC \\
\hline G0.G1 & 46.59 & 54.13 & 61.26 & 59.89 \\
\hline S & 46.37 & 30.30 & 33.63 & 25.10 \\
\hline G2M & 7.05 & 15.56 & 5.10 & 15.10 \\
\hline
\end{tabular}
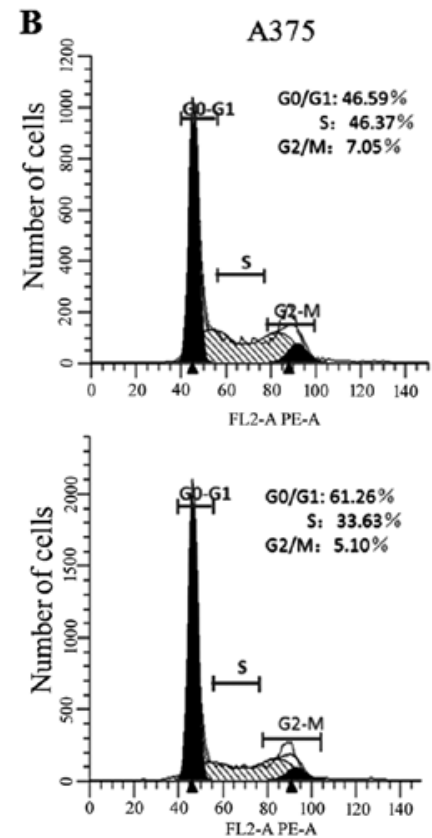
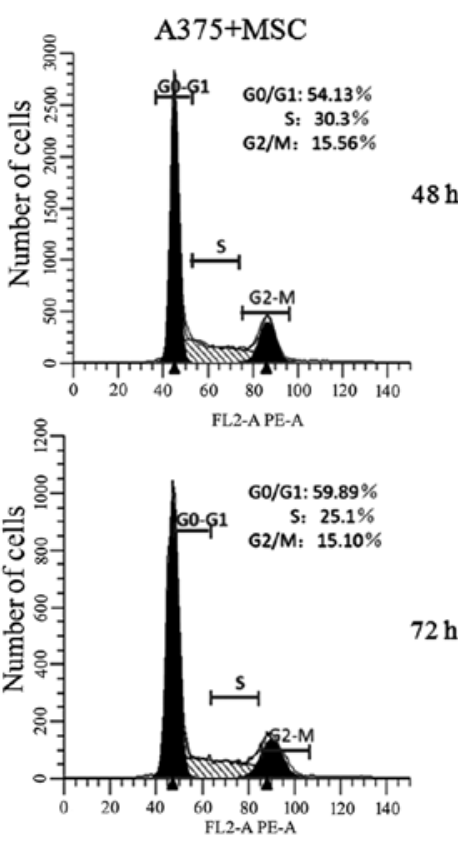

Figure 2. Proliferation and cell cycle assay by flow cytometry. (A) Proliferation assay by MTT. (B) Cell cycle detection (C). Cell cycle analysis. ${ }^{* *} \mathrm{P}<0.01$ vs. the control.
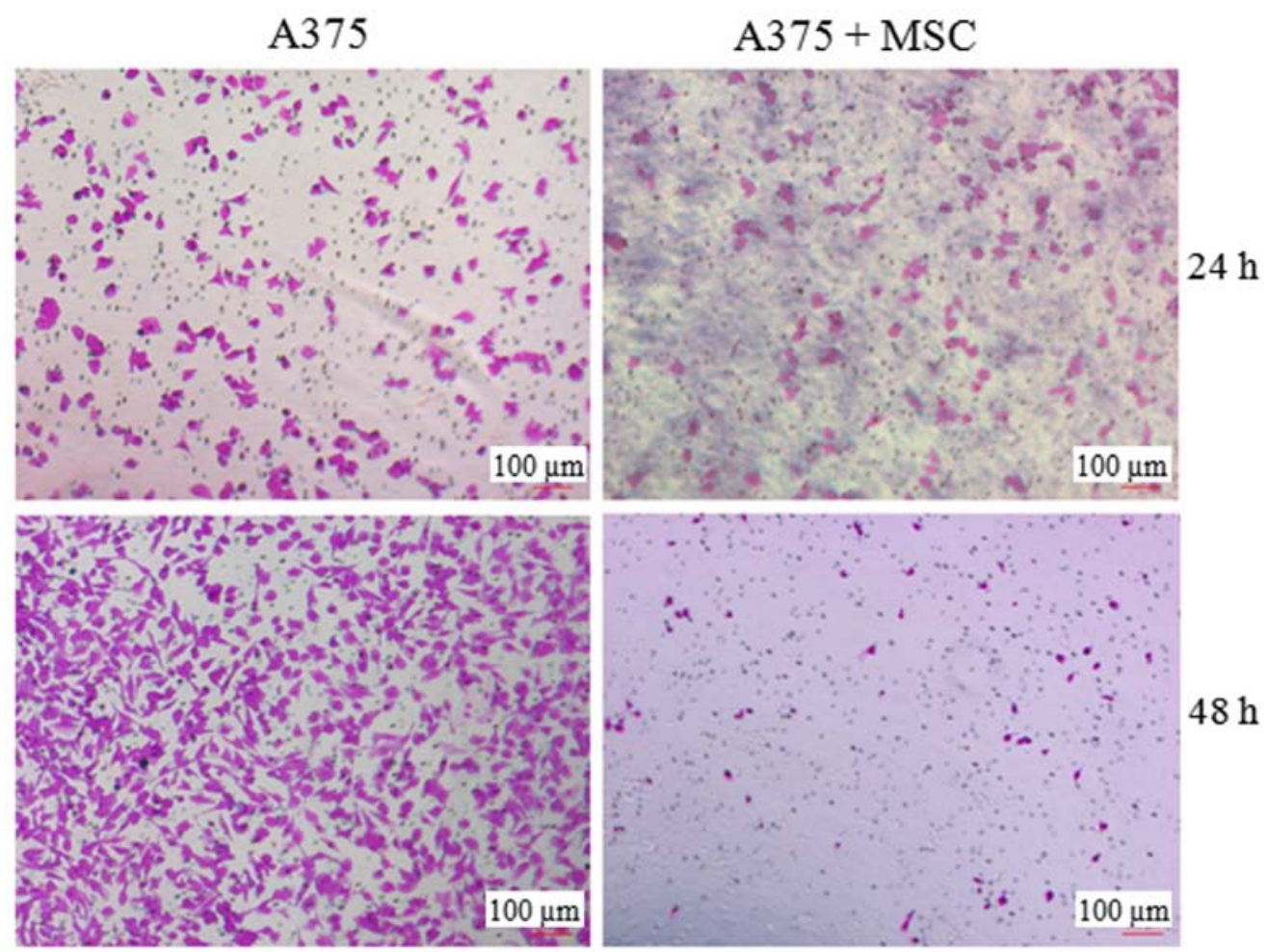

Figure 3. Detection of the invasion of A375 cells co-cultured with UCMSCs. Magnification, x100. UCMSCs, umbilical cord-derived mesenchymal stem cells.

UCMSCs inhibit the expression of several AKT/STAT3/ $P I 3 K / m T O R$ pathway components in A375 cells. To identify the mechanism by which the functions of A375 cells were attenuated by UCMSCs, western blotting was performed to detect the protein expression of several important kinases at 48 and $72 \mathrm{~h}$ post co-culture. We found that all tested kinases (AKT/p-AKT, PI3K/p-PI3K, STAT3/p-STAT3, ERK/p-ERK and mTOR), except ERK $(\mathrm{P}<0.05)$, demonstrated no significant variation between the A375 monoculture and A375+MSC co-culture groups at $48 \mathrm{~h}$ (Fig. 4). Following $72 \mathrm{~h}$ of co-culture, UCMSCs led to the downregulation of the expression of AKT $(\mathrm{P}<0.05)$, p-AKT $(\mathrm{P}<0.01)$, p-PI3K $(\mathrm{P}<0.05)$, -STAT3 $(\mathrm{P}<0.01)$, ERK $(\mathrm{P}<0.01)$, p-ERK $(\mathrm{P}<0.05), \operatorname{mTOR}(\mathrm{P}<0.05)$ and p-mTOR $(\mathrm{P}<0.05)$ (Fig. 4). These results indicated the important role of the AKT/STAT3/PI3K/mTOR pathway in the regulation of the function of A375 cells by UCMSCs. 

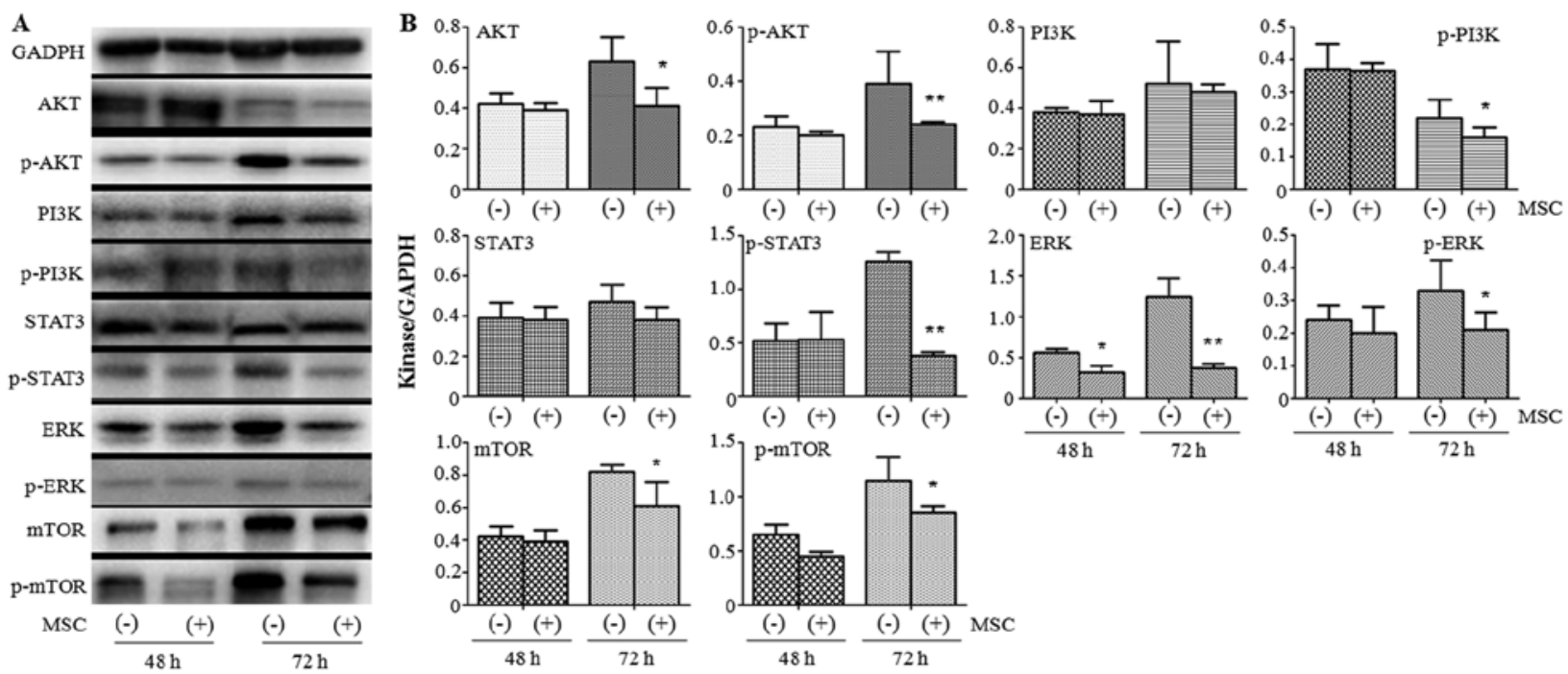

Figure 4. Expression of kinases of A375 cells co-cultured with UCMSCs. (A) Western blot analysis. (B) Data analysis. ${ }^{*} \mathrm{P}<0.05,{ }^{* *} \mathrm{P}<0.01$ vs. the control. UCMSCs, umbilical cord-derived mesenchymal stem cells.
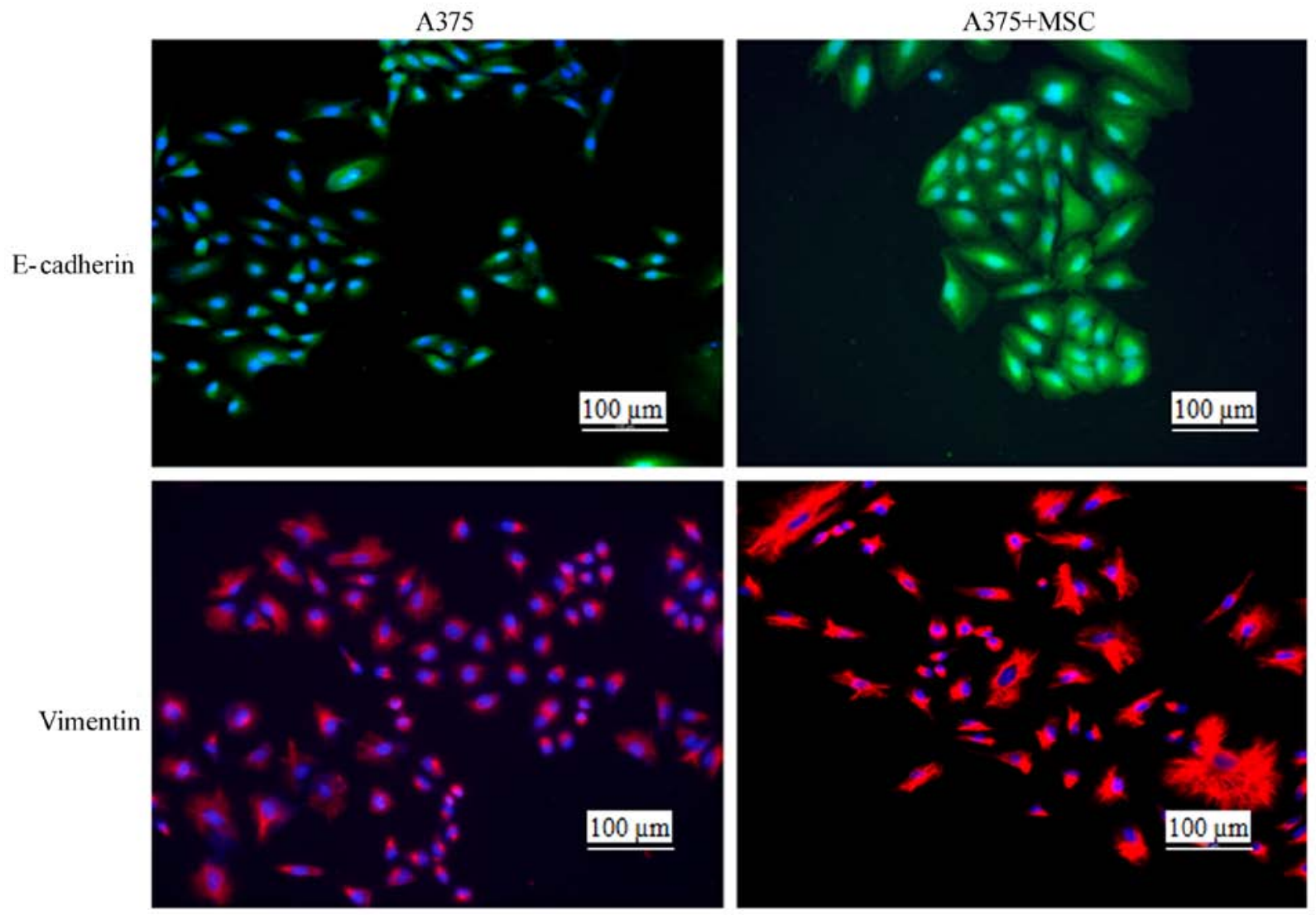

Figure 5. Cytoskeletal rearrangement of the A375 cells co-cultured with UCMSCs. Magnification, x100. UCMSCs, umbilical cord-derived mesenchymal stem cells.

UCMSCs promote cytoskeletal remodeling in A375 cells. The invasion of malignant tumor cells involves decreased adherence and enhanced migration properties, and these changes are part of the epithelial-mesenchymal transition (EMT) (16). During the EMT process, cytoskeletal molecules (including E-cadherin, N-cadherin, laminin and vimentin) play important regulatory roles (17). In the present study, we detected the occurrence of cytoskeletal rearrangement by staining for E-cadherin and vimentin at $48 \mathrm{~h}$ post co-culture with UCMSCs. The results indicated that the cytoskeletal rearrangement was obvious in the A375+MSC co-culture group compared with the A375 monoculture group (Fig. 5), indicating that in the presence of UCMSCs, the invasion of A375 cells may be regulated by certain cytoskeletal molecules. 


\section{Discussion}

In the present study, we co-cultured melanoma A375 cells with MSCs isolated from umbilical cord to study the role of UCMSCs in regulating the functions of A375 cells. Although UCMSCs had no effect on the cell cycle of A375 cells, this study clearly indicated that UCMSCs inhibited the cell proliferation and invasion, as well as induced the apoptosis of A375 cells. In the mechanistic analysis, the expression levels of AKT/PI3K/STAT3/mTOR pathway components were downregulated following UCMSC co-culture. Furthermore, marked cytoskeletal rearrangement was observed in the A375+UCMSC co-culture group. Collectively, these results confirmed that UCMSCs can exert an antitumor effect on A375 melanoma cells.

The effect of MSCs on malignant tumor cells remains controversial. The reported discrepancies may be due to the heterogeneity of MSCs or the timing and dose of MSC treatments, or the involvement of other unknown molecules and mechanisms. Liu et al (18) reported that UCMSCs could inhibit the growth of human cholangiocarcinoma HCCC-9810 cells in xenograft models, and that UCMSC-conditioned medium suppressed cell proliferation (inhibition rate: 6.21 vs. $49.86 \%$ ) and induced cell apoptosis (9.3 vs. $48.1 \%$ ) in a dose- and time-dependent manner. In an immunoblot analysis, the same authors also found that p-PDK1, p-AKT, $\beta$-catenin, cyclin-D1 and c-Myc mediated the functional changes of HCCC-9810 cells induced by UCMSCs (18). In another study, pancreatic cancer cells were co-implanted with MSCs in NOD/SCID mice, and it was demonstrated that MSC-derived myofibroblast-like cells could maintain the stemness of tumor-initiating stem cells among pancreatic cancer cells. The mechanistic analysis indicated that the Notch-signaling pathway appeared to contribute to the regulation of stemness by MSCs (13). Wang et al (19) explored the effect of the fusion of MSCs with esophageal carcinoma cells, and observed that MSCs markedly decreased tumor cell growth, increased apoptosis and suppressed tumorigenicity. They also observed that the expression of DUSP6/MKP3 in the MAPK pathway increased and the exogenous overexpression confirmed the growth suppression. Sun et al (20) engineered MSCs stably transfected with TNF-related apoptosis-inducing ligand (TRAIL) and co-cultured them with hepatoblastoma HepG2 cells directly, or applied MSC-conditioned media to the HepG2 cells. The survival rate of HepG2 cells was markedly decreased by the co-culture conditions, and TRAIL was indicated to serve an important role in promoting cell death. All of these studies revealed the uncertainty regarding the effect of MSCs on malignant tumors.

However, the present study clearly demonstrated the antitumor effect of MSCs on A375 melanoma cells in a co-culture system. We not only observed inhibition of proliferation, induction of apoptosis and suppression of invasion, but also further investigated the possible mechanism of the MSC-mediated antitumor effect. Certain important kinases (including AKT, PI3K and STAT3) were downregulated in A375 cells upon MSC co-culture, and this finding was consistent with previous research. Of note was the observation of the rearrangement of the cytoskeleton of A375 cells co-cultured with MSCs. Based on this, we hypothesized that MSCs could inhibit tumor invasiveness by regulating the EMT. However, further research is required to confirm this hypothesis. The limitation of the present study was that only one cell line was included, since this was just a preliminary study concerning the role of UCMSCs in regulating malignant tumor functions. Our following study will include tumor cell lines from lung, cervical, breast and prostate cancer.

\section{Acknowledgements}

The authors thank the professor Li Zhang (Laboratory of Pathology, West China Hospital, Sichuan University) for his continuous support to this work.

\section{Funding}

The present study was supported by Sichuan Province Science and Technology Support Program (nos. 2014SZ0206 and 2014SZ0001).

\section{Availability of data and materials}

The datasets used during the study are available from the corresponding author upon reasonable request.

\section{Authors' contributions}

WW carried out fluorescence detection, statistical analysis and drafted the manuscript. LL was responsible for completing all the cell related experiments. FC carried out PCR experiments and western blot analysis. YY conceived of the study and participated in its design and coordination and helped to draft the manuscript. All authors read and approved the manuscript and agree to be accountable for all aspects of the research in ensuring that the accuracy or integrity of any part of the work are appropriately investigated and resolved.

\section{Ethics approval and consent to participate}

All experimental protocols have been approved by the Ethics Committee of West China Hospital, Sichuan University (Chengdu, China).

\section{Consent for publication}

Not applicable.

\section{Competing interests}

The authors state that they have no competing interests.

\section{Authors' information}

WW is a professor of the Department of Pathology, West China Second University Hospital, Chengdu. LL is a laboratory director at the Laboratory of Pathology, West China Hospital, Sichuan University, Chengdu. FC is an assistant laboratory director at the Laboratory of Pathology, West China Hospital, Sichuan University, Chengdu. YY is a professor of the Department of Medical Oncology, Cancer Center of West China Hospital, Sichuan University, Chengdu, P.R. China. 


\section{References}

1. Pittenger MF, Mackay AM, Beck SC, Jaiswal RK, Douglas R Mosca JD, Moorman MA, Simonetti DW, Craig S and Marshak DR: Multilineage potential of adult human mesenchymal stem cells. Science 284: 142-147, 1999.

2. Phinney DG and Prockop DJ: Concise review: Mesenchymal stem/multipotent stromal cells: The state of transdifferentiation and modes of tissue repair-current views. Stem Cells 25: 2896-2902, 2007.

3. Bassi EJ, Aita CA and Camara NO: Immune regulatory properties of multipotent mesenchymal stromal cells: Where do we stand? World J Stem Cell 3: 1-8, 2011.

4. Shi M, Liu ZW and Wang FS: Immunomodulatory properties and therapeutic application of mesenchymal stem cells. Clin Exp Immunol 164: 1-8, 2011.

5. Sensebe L, Krampera M, Schrezenmeier H, Bourin P and Giordano R: Mesenchymal stem cells for clinical application. Vox Sang 98: 93-107, 2010.

6. Sun L, Wang D, Liang J, Zhang H, Feng X, Wang H, Hua B Liu B, Ye S, Hu X, et al: Umbilical cord mesenchymal stem cell transplantation in severe and refractory systemic lupus erythematosus. Arthritis Rheum 62: 2467-2475, 2010.

7. Wang HS, Shu JF, Shen WS, Hsu HC, Chi TC, Chen CP, Huang SW, Shyr YM, Tang KT and Chen TH: Transplantation of insulin-producing cells derived from umbilical cord stromal mesenchymal stem cells to treat NOD mice. Cell Trnsplant 20: 455-466, 2011

8. Zhang Z, Lin H, Shi M, Xu R, Fu J, Lv J, Chen L, Lv S, Li Y, Yu S, et al: Human umbilical cord mesenchymal stem cells improve liver function and ascites in decompensated liver cirrhosis patients. J Gastroenterol Hepaol 27 (Suppl 2): S112-S120, 2012.

9. Wang J, Yin Z, Wen H, Wang L and Chen Z: Application of umbilical cord mesenchymal stem cells in cerebral palsy treatment: Report of 51 cases. Chinse Gen Pract 14: 2446-2447, 2011.

10. Liu Y, Mu R, Wang S, Long L, Liu X, Li R, Sun J, Guo J, Zhang X, Guo J, et al: Therapeutic potential of human umbilical cord mesenchymal stem cells in the treatment of rheumatoid arthritis. Arthritis Res Ther 12: R210, 2010.
11. Ma $\mathrm{Y}$, Hao $\mathrm{X}$, Zhang $\mathrm{S}$ and Zhang $\mathrm{J}$ : The in vitro and in vivo effects of human umbilical cord mesenchymal stem cells on the growth of breast cancer cells. Breast Cancer Res Treat 133: 473-485, 2012.

12. Matsuzuka T, Rachakatla RS, Doi C, Maurya DK, Ohta N, Kawabata A, Pyle MM, Pickel L, Reischman J, Marini F, et al: Human umbilical cord matrix-derived stem cells expressing interferon-beta gene significantly attenuate bronchioloalveolar carcinoma xenografts in SCID mice. Lung Cancer 70: 28-36, 2010.

13. Kabashima-Niibe A, Higuchi H, Takaishi H, Masugi Y, Matsuzaki Y, Mabuchi Y, Funakoshi S, Adachi M, Hamamoto Y, Kawachi S, et al: Mesenchymal stem cells regulate epithelial-mesenchymal transition and tumor progression of pancreatic cancer cells. Cancer Sci 104: 157-164, 2013.

14. Zhang T, Lee YW, Rui YF, Cheng TY, Jiang XH and Li G: Bone marrow-derived mesenchymal stem cells promote growth and angiogenesis of breast and prostate tumors. Stem Cell Res Ther 4: 70, 2013 .

15. Yang Y, Wang Y, Li L, Chen F and Zhang P: Activation of the Toll-like receptor 8 pathway increases the immunogenicity of mesenchymal stem cells from umbilical cord. Mol Med Rep 16: 2061-2068, 2017

16. Thiery JP: Epithelial-mesenchymal transition in tumor progression. Nat Rev Cancer 2: 442-454, 2002.

17. Kalluri R and Weinberg RA: The basics of epithelial-mesenchymal transition. J Clin Invest 119: 1420-1428, 2009.

18. Liu J, Han GQ, Liu H and Qin C: Suppression of cholangiocarcinoma cell growth by human umbilical cord mesenchyaml stem cells: A possible role of wnt and Akt signaling. PLoS One 8: e62844, 2013.

19. Wang Y, Fan H, Zhou B, Ju Z, Yu L, Guo L, Han J and Lu S: Fusion of human umbilical cord mesenchymal stem cells with esophageal carcinoma cells inhibits the tumorigenecity of esophageal carcinoma cells. Int J Oncol 40: 370-377, 2012.

20. Sun XY, Nong J, Qin K, Lu H, Moniri MR, Dai LJ and Warnock GL: MSC (TRAIL)-mediated HepG2 cell death in direct and indirect co-culture. Anticancer Res 31: 3705-3712, 2011. 